\title{
PERSPECTIVES
}

OPINION

\section{Models matter: the search for an effective Staphylococcus aureus vaccine}

\section{Wilmara Salgado-Pabón and Patrick M. Schlievert}

Abstract | Staphylococcus aureus is a highly successful bacterial pathogen owing to its abundance of cell surface and secreted virulence factors. It is estimated that $30 \%$ of the population is colonized with S. aureus, usually on mucosal surfaces, and methicillin-resistant $S$. aureus is a major public health concern. There have been multiple attempts to develop an $S$. aureus vaccine using one or more cell surface virulence factors as antigens; all of these vaccine trials have failed. In this Opinion article, we suggest that an over-reliance on rodent models and a focus on targeting cell surface components have been major contributing factors to this failure.

Vaccination is widely recognized as one of the most cost-effective public health interventions. Early vaccine development efforts that targeted bacterial pathogens resulted in vaccines for Clostridium tetani, Corynebacterium diphtheriae and Bordetella pertussis, and later efforts resulted in vaccines that targeted Streptococcus pneumoniae, Haemophilus influenzae and Neisseria meningitidis. Most of these vaccines involved a single antigenic target - either a toxin or the capsule - that, when targeted, provided immune protection. The development of vaccines for most remaining bacterial pathogens, including Staphylococcus aureus, is more difficult, as the pathogenesis of these organisms is multifaceted.

S. aureus is the most important cause of serious infectious diseases, such as toxic shock syndrome (TSS), pneumonia, sepsis and infective endocarditis (FIG. 1), in the United States ${ }^{1}$. The life-threatening illnesses that are associated with $S$. aureus infection are dependent both on cell surface and on secreted virulence factors ${ }^{2,3}$. As the emergence of multidrug-resistant $S$. aureus strains is predicted to continue, efforts have focused on disease prevention via vaccination or immunotherapeutic approaches. As a minimum, it is desirable to develop vaccines that protect against life-threatening $S$. aureus infections, but it is hoped that vaccines could be developed that protect against all $S$. aureus infections, including the many soft tissue infections, such as abscesses and surgical site infections, that are associated with $S$. aureus. The vaccine approaches that have been used so far have all involved targeting cell surface components, including the polysaccharide capsule, the extracellular polysaccharides that are important for biofilm formation, the cell wall-associated proteins that recognize adhesive matrix molecules and aid in surface attachment and colonization, or ATP-binding cassette (ABC) transporters, which have a role in nutrient uptake and drug resistance. However, none of these immunization strategies has succeeded in human trials (BOX 1). The latest S. aureus vaccine failure ${ }^{4}$ emphasizes the need to address the reasons for these failures and provides an opportunity to correct them.

Recent discussions on the failure of staphylococcal vaccines have focused on our lack of understanding of $S$. aureus pathogenesis in humans $s^{5-7}$, on the large range of virulence factors that are produced by $S$. aureus (many of which have redundant functions) and their contributions to $S$. aureus pathogenesis ${ }^{5,8-11}$, as well as on the nature of protective immunity against $S$. aureus infection $^{8,12-14}$. There seems to be a consensus among investigators that the multiplicity and redundancy of $S$. aureus virulence factors creates an enormous challenge for the development of an effective vaccine and that the failure of vaccine development so far is a result of not knowing the correct combination of antigens or of using a range of antigens that is too narrow ${ }^{7,9,10}$. Furthermore, some argue that not knowing the correlates of immune protection is the greatest obstacle to vaccine development ${ }^{11,13,14}$. In addition, the protective role of antibodies in immune defence against $S$. aureus infections has also been questioned, and it has been argued that opsonic $S$. aureus-specific antibodies already exist in humans and are not sufficient to provide protection from infection, and an antibody-based vaccine is therefore unlikely to succeed ${ }^{14,15}$.

More than 100 years have passed since the discovery of $S$. aureus and the diseases that it causes, and we still know relatively little about $S$. aureus pathogenesis ${ }^{3}$. As Projan et al. wrote, "little knowledge is a dangerous thing" ". However, the development of useful vaccines and immunotherapeutic agents to target $S$. aureus infections depends on a correct understanding of the molecular pathogenesis of $S$. aureus. This requires the use of appropriate animal models. Mice have been the animal models of choice for most pathogenesis studies, owing to their low cost. However, the most important consideration when selecting a model system for preclinical studies should be whether or not it closely mimics human infections. So far, mouse models of S. aureus infection have failed to predict human outcomes in clinical trials ${ }^{8,12}$. Although many investigators concede that mouse models are imperfect ${ }^{5-7,10,12,14}$, in practice, most investigators continue to dismiss these inadequacies and still expect these models to shed light on crucial aspects of $S$. aureus pathogenesis ${ }^{16}$ and mechanisms of immune protection against $S$. aureus infection ${ }^{8,17}$.

It is also crucial to recognize that $S$. aureus causes serious illnesses, most notably menstrual and non-menstrual TSS ${ }^{18}$, lethal pneumonia ${ }^{19}$, infective endocarditis 


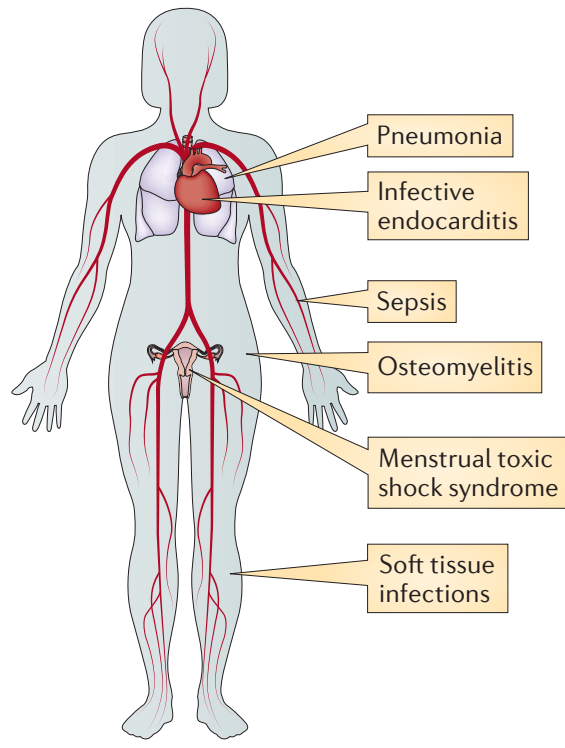

Figure 1 | Diseases caused by Staphylococcus aureus. S. aureus causes life-threatening diseases, including pneumonia, infective endocarditis, toxic shock syndrome and sepsis, as well as minor diseases such as soft tissue infections.

and sepsis ${ }^{20}$, via massive immune system activation and dysregulation, and the lethality of these diseases is dependent on the effects of superantigens (SAgs), such as TSS toxin 1 (TSST1), staphylococcal enterotoxin B (SEB), SEC, and cytotoxins, such as $\alpha$-toxin, on the cardiovascular system (reviewed in REF. 19). Physiologists recognize that the cardiovascular physiology of mice does not resemble that of humans ${ }^{21}$, and in our opinion, this is a major drawback of mouse models of $S$. aureus infection. By contrast, the cardiovascular physiology of rabbits closely resembles that of humans ${ }^{22,23}$. Rabbits and humans also respond similarly to SAgs and cytotoxins, whereas mice respond differently ${ }^{24}$. Although rabbits are more difficult to work with, owing to the cost, the lack of inbred strains and greater government regulatory control, their immune and cardiovascular systems respond to $S$. aureus infection in a manner that is more comparable with that of humans.

In this Opinion article, we discuss the soundness of targeting $S$. aureus surface components, compared with secreted factors, as a vaccine strategy, as well as the use of mouse models to study $S$. aureus pathogenesis and vaccine outcomes.

\section{Targeting the S. aureus surface}

Most $S$. aureus vaccines or immunotherapies that have been tested or that are in development target surface components. The failure of the V710 vaccine (Merck) (which targeted the cell wall-anchored iron-responsive surface determinant B (IsdB)), in which vaccinated patients who developed an S. aureus infection were fivefold more likely to die than unvaccinated patients who developed an $S$. aureus infection ${ }^{4}$, took the field by surprise, and investigators have struggled to make sense of these findings.

However, it is well-established that cell surface virulence factors facilitate host colonization via a combination of cell attachment and avoidance of the innate immune system. S. aureus uses a range of these virulence factors (for example, fibronectin-binding proteins and clumping factors) to aggregate, forming large bacterial clumps that are protected by a capsule and 'decorated' with host factors ${ }^{7,25,26}$. We hypothesize that antibodies that are directed against surface components further promote $S$. aureus aggregation. These large aggregates of bacteria, antibodies and other host factors in the blood may not be cleared by the host and could become trapped in various tissues (particularly in the lungs, but also in skin, the kidneys, the liver and joints) where they would fix complement, recruit granulocytes and other phagocytes and induce the release of pro-inflammatory mediators, as well as potentially causing septic emboli. Should this prove to be the case, then the expected consequences are tissue damage, ischaemia and abscess formation, which would lead to multi-organ failure and shock. Hence, in our opinion, targeting staphylococcal surface components does not seem to be a sound vaccine strategy.

Scientific evidence to support our hypothesis comes from published vaccination studies that were carried out in the Gram-positive pathogen Enterococcus faecalis and from recent studies with S. aureus in the rabbit model of infective endocarditis. It is important to consider that S. aureus and enterococci both form aggregates and both cause infective endocarditis. The enterococcal aggregation substance (AS), as the name suggests, promotes the formation of large bacterial aggregates and mediates attachment to eukaryotic cells ${ }^{27,28}$. AS is a major contributor to E. faecalis pathogenesis in rabbit models of infective endocarditis and sepsis ${ }^{29,30}$. Vaccination with heat-killed or gentamicin-treated aggregation substance-expressing $\left(\mathrm{AS}^{+}\right)$E. faecalis, followed by challenge with either $\mathrm{AS}^{+}$or $\mathrm{AS}^{-}$organisms, resulted in endocarditis vegetations and increased lethality in $\mathrm{AS}^{+}$ E. faecalis-infected rabbits ${ }^{27}$. AS-specific antibodies were shown to promote the aggregation of $E$. faecalis in vitro, which suggests that antibody-mediated hyperaggregation contributes to poorer disease outcomes and increased lethality in vaccinated rabbits. However, treatment of rabbits with monovalent, AS-specific antibody fragments (IgG Fabs), which had been shown to prevent aggregation of $\mathrm{AS}^{+} E$. faecalis in vitro, effectively decreased vegetation size, reduced bacterial recovery from vegetations and increased survival when challenged with $\mathrm{AS}^{+}$E. faecalis ${ }^{27}$.

A similar effect was recently observed with $S$. aureus ${ }^{19}$. Rabbits were vaccinated with an $S$. aureus multivalent surface antigen preparation that was enriched for Isds, and were challenged intravenously with a sublethal dose of S. aureus MW2. Vaccinated rabbits succumbed to infection within 6 hours of MW2 challenge, whereas unvaccinated rabbits, albeit sick, survived to day 4 (REF. 19). Hence, these studies suggest that $E$. faecalis and $S$. aureus share the property of increased disease severity in the presence of antibodies that target their surface components. In our opinion, the increased incidence of multi-organ failure and death in the V710-vaccinated human cohort that acquired $S$. aureus bacteraemia ${ }^{4}$ could be explained by a similar mechanism. These effects are quite dramatic and require further investigation in S. aureus; however, mouse models of $S$. aureus infection do not reproduce these lethal effects.

\section{Mice do not mimic humans}

Resistance to lipopolysaccharide, cytotoxins and SAgs. Mouse immune and cardiovascular systems respond differently to bacterial toxins, compared with human and rabbit systems, which partly explains their resistance to the lethal effects of lipopolysaccharide (LPS; also known as endotoxin), SAgs and cytotoxins (TABLE 1). The lethal dose of LPS in humans is approximately $0.01 \mu \mathrm{g}$ per $\mathrm{kg}$ (REF. 31), and the lethal dose in rabbits is approximately $0.5 \mu \mathrm{g}$ per $\mathrm{kg}$ (REF. 32). However, in commonly used laboratory mice, the LPS lethal dose is $>80,000 \mu \mathrm{g}$ per $\mathrm{kg}$ (REFS 33,34). The lethal doses of cytotoxins, notably a-toxin, in humans are unknown, but $\alpha$-toxin is considered to be highly lethal when administered intravenously, as evidenced in the Bundaberg, Australia, disaster in which 12 of 21 recipients of a diphtheria toxoid vaccine developed fatal disease that was associated with the production of $\alpha$-toxin by an $S$. aureus contaminant ${ }^{35}$. In rabbits (specifically, Dutch-belted rabbits), $0.005 \mu \mathrm{g}$ per $\mathrm{kg}$ of a-toxin injected intravenously kills in 24 hours and $0.5 \mu \mathrm{g}$ per $\mathrm{kg}$ kills in 1 minute ${ }^{36}$. 


\section{Box 1 | Immunization: mouse versus human}

So far, two Staphylococcus aureus vaccines (StaphVAX (Nabi Biopharmaceuticals) and V710 (Merck)) and five passive immunization preparations (Pagibaximab (Biosynexus, Glaxo Smith Kline), tefibazumab (Aurexis; Inhibitex), Veronate (Inhibitex), Aurograb (Novartis) and AltaStaph (Nabi Biopharmaceuticals)) have completed clinical trials. All seven vaccines targeted S. aureus surface components.

Pagibaximab is a humanized monoclonal antibody that is targeted against lipoteichoic acid (LTA). LTA is an important component of the Gram-positive cell wall and is essential for its integrity and for bacterial survival ${ }^{84}$. Pagibaximab was tested in very low birthweight neonates for the prevention of staphylococcal sepsis ${ }^{85,86}$. In both studies, Pagibaximab showed a positive trend, but the results were not conclusive. A larger study was recently completed, and Pagibaximab failed to reduce staphylococcal sepsis in this population ${ }^{87}$.

StaphVAX is a bivalent vaccine that is composed of capsular polysaccharide 5 (CP5) and CP8 individually conjugated to recombinant exoprotein $\mathrm{A}(\mathrm{rETA})$ from Pseudomonas aeruginosa ${ }^{88}$. $\mathrm{CP} 5$ and $\mathrm{CP} 8$ are highly conserved and are produced by most pathogenic $\mathrm{S}$. aureus strains ${ }^{89-92}$. CP5 increased bacterial virulence in a mouse model of bacteraemia and arthritis, compared with a CP5-deficient strain ${ }^{93}$. Vaccination of mice with CP5-rETA provided protection against a lethal challenge ${ }^{94}$. In humans, StaphVAX failed to reduce the incidence of S. aureus bacteraemia in patients undergoing haemodialysis ${ }^{95.96}$.

AltaStaph comprises pooled hyperimmune polyclonal antibodies from healthy individuals who have been vaccinated with StaphVAX ${ }^{97}$. Treatment of mice with AltaStaph significantly reduced bacteraemia and deep tissue infections after sublethal challenge ${ }^{94}$. Treatment of rats with rabbit CP5-rETA-specific antibodies lowered the prevalence of infective endocarditis and decreased bacterial counts in vegetations, blood and kidneys ${ }^{98}$. In humans, AltaStaph failed to reduce the incidence of S. aureus bacteraemia in low birthweight neonates ${ }^{97}$ or to improve the outcome of patients with persistent bacteraemia ${ }^{99}$.

Tefibazumab (which comprises humanized monoclonal antibodies) and Veronate (which is a pooled human hyperimmune preparation) target clumping factor $\mathrm{A}$ (ClfA). ClfA is a cell wall protein that binds to fibrinogen and fibrin and promotes bacterial clumping in plasma and adherence to blood clots. Mutational analysis provided evidence for the role of ClfA in S. aureus pathogenesis in a mouse model of septic arthritis and a rat model of indwelling catheter-infective endocarditis ${ }^{100,101}$. Vaccination of mice with recombinant truncated ClfA led to less severe arthritis after $S$. aureus challenge. In humans, tefibazumab failed to prevent $S$. aureus bacteraemia relapse, complications or death ${ }^{102}$; Veronate failed to reduce the incidence of late-onset sepsis in premature neonates ${ }^{103}$.

Aurograb is a single-chain fragment of the immunoglobulin variable region that is specific for the S. aureus ATP-binding cassette (ABC) transporter GrfA. Human sera from 26 patients with epidemic methicillin-resistant S. aureus 15 (EMRSA-15) septicaemia contained high antibody titres against three different $S$. aureus $A B C$ transporter peptides ${ }^{104}$. In a mouse sepsis model, treatment with human antibodies to the individual peptides significantly reduced the recovery of EMRSA-15 from the kidneys, liver and spleen ${ }^{105}$. Aurograb failed to show efficacy when tested as an add-on therapy in patients with severe, deep-seated staphylococcal infections ${ }^{6,106}$.

V710 is a monovalent vaccine that is composed of the iron-responsive surface determinant B (IsdB), which is a cell wall-anchored protein that is conserved among diverse S. aureus strains and is expressed during iron limitation ${ }^{4,107}$. The vaccine provided protection against nasal carriage in a cotton rat model, increased survival in a mouse sepsis model and induced high-level antibody titres in mice ${ }^{16,108,109}$. In humans, V710 failed to reduce the incidence of post-operative S. aureus bacteraemia and major chest wound infection, and it was associated with an increased incidence of multi-organ dysfunction and increased mortality in members of the vaccinated cohort who developed S. aureus bacteraemia ${ }^{4,110}$.

By contrast, in $\mathrm{BALB} / \mathrm{c}$ mice, the lethal dose of $\alpha$-toxin is $>200,000 \mu \mathrm{g}$ per $\mathrm{kg}$ (P.M.S, unpublished observations).

The difference between mice, rabbits and humans in their susceptibility to SAgs is even more striking. Humans who have been injected intravenously with SEA at doses as low as $0.001 \mu \mathrm{g}$ per $\mathrm{kg}$ develop fever and hypotension (which are symptoms of TSS) ${ }^{37}$. In rabbits, intrapulmonary inoculation of TSST1 or continuous exposure to TSST1 at $0.05 \mu \mathrm{g}$ per $\mathrm{kg}$ implanted subcutaneously in mini-osmotic pumps causes $100 \%$ lethality ${ }^{24}$. In mice, continuous exposure to TSST1 at $4,000,000 \mu \mathrm{g}$ per kg in mini-osmotic pumps is not letha ${ }^{24}$. To induce susceptibility to SAgs in mice, investigators have used potent sensitizing agents, such as the hepatotoxin D-galactosamin ${ }^{24}$; however, these mice develop what seems to be fulminant liver failure. This condition is not observed in humans or rabbits with TSS ${ }^{38,39}$. Therefore, standard mouse models have reduced sensitivity to LPS, cytotoxins and SAgs.
Attenuated virulence. A comparison of the virulence of representative isolates of S. aureus USA200, USA300 and USA 400 clonal types has been carried out in animal models of pneumonia, sepsis and infective endocarditis. The USA200 isolates colonize human mucosal surfaces and are frequently associated with life-threatening infections, such as post-influenza pneumonia, surgical wound infections, infective endocarditis, sepsis and TSS ${ }^{40-43}$. USA200 isolates are also associated with almost all cases of menstrual TSS, which develops in otherwise healthy females ${ }^{18}$. USA200 strains usually produce $\alpha$-toxin at low levels $(0.05-5.0 \mu \mathrm{g} \mathrm{per} \mathrm{ml})^{36,44}$ and TSST1 at high levels (5-20 $\mu \mathrm{g}$ per ml in liquid culture and up to $16,000 \mu \mathrm{g}$ per $\mathrm{ml}$ in biofilms) or $\alpha$-toxin, TSST1 and SEC, which is also produced at high levels (up to $100 \mu \mathrm{g}$ per $\mathrm{ml}$ in liquid culture $)^{45}$. In the rabbit pneumonia model, USA200 isolates cause $90 \%$ lethality in 48 hours at doses of $6.5 \times 10^{8}$ colony-forming units (CFUs) per $\mathrm{kg}^{46}$. Furthermore, in a rabbit infective endocarditis or sepsis model, USA200 isolates effectively cause lethal sepsis, with the $\mathrm{LD}_{50}$ (dose lethal to $50 \%$ of animals tested) calculated at $0.3-1.5 \times 10^{8}$ CFUs per $\mathrm{kg}$ (REF. 47). By contrast, in the mouse pneumonia model, USA200 strains inoculated at a dose of $1.0 \times 10^{10} \mathrm{CFUs}$ per $\mathrm{kg}$ fail to induce lethality in a 48 hour time period and exhibit a greatly reduced capacity to induce lethality in a sepsis mode ${ }^{48}$.

USA300 isolates are commonly community-associated methicillin-resistant S. aureus (CA-MRSA) strains, which are frequently associated with skin and soft tissue infections and necrotizing pneumonia ${ }^{49}$. The USA300 LAC strain produces $\alpha$-toxin at high levels $(\sim 150-500 \mu \mathrm{g} \text { per } \mathrm{ml})^{50}$ and the SAgs SE-like X (SEl-X) ( 200 ng per ml in liquid culture), SEl-Q and SEl-K at low levels $(<30 \mathrm{ng} \mathrm{per} \mathrm{ml})^{20}$. In the rabbit pneumonia model, LAC inoculated at $6.5 \times 10^{8}$ CFUs per kg induces $90 \%$ lethality 24 hours post-infection; whereas, in the mouse pneumonia model, $80-90 \%$ lethality is reported with an inoculum that is tenfold higher on a per-kilogram basis than that used for rabbits $^{51}$. Furthermore, an intravenous dose of $6.5 \times 10^{8} \mathrm{CFUs}$ per kg in rabbits results in $100 \%$ mortality in $<18$ hours, whereas a five-times higher dose is required to obtain comparable lethality $(90 \%)$ in mice in 24 hours $^{44,52}$.

The USA400 strain MW2 is a CA-MRSA that was isolated from a child with lethal necrotizing pneumonia. MW2 produces moderate amounts of $\alpha$-toxin $(\sim 50 \mu \mathrm{g}$ per $\mathrm{ml})^{53}$ and encodes an array of SAgs, 


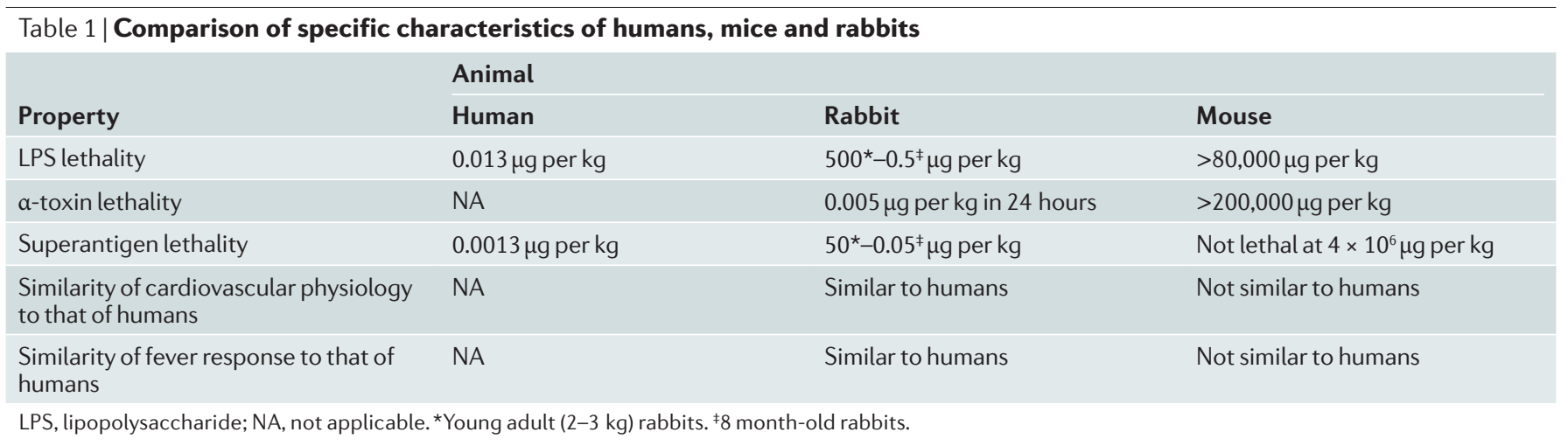

including SEC and SEl-X. SEC is the dominant SAg in MW2 - it is produced at $\sim 100 \mu \mathrm{g}$ per $\mathrm{ml}$ in liquid culture - and is required for the development of necrotizing pneumonia and lethal sepsis in rabbits ${ }^{46}$. MW2 doses of $6.5 \times 10^{8}$ CFUs per kg are $100 \%$ lethal in rabbits 48 hours after infection. Strikingly, a tenfold increase in the MW2 dose on a per-kilogram basis results in only $20 \%$ lethality in mice and $36 \%$ lethality in rats in the same 48 hour period ${ }^{54,55}$.

Overall, the mouse models seem to mostly reflect the effect of high levels of a-toxin production, as they completely lack sensitivity to SAgs and to lower levels of a-toxin (FIG. 2). Therefore, S. aureus strains such as MN8 seem to be avirulent in mice (as TSST1 has no effect and this strain produces low levels of $\alpha$-toxin), MW2 seems to have greatly reduced lethality (although it produces high levels of SEC and moderate levels of $\alpha$-toxin) and LAC
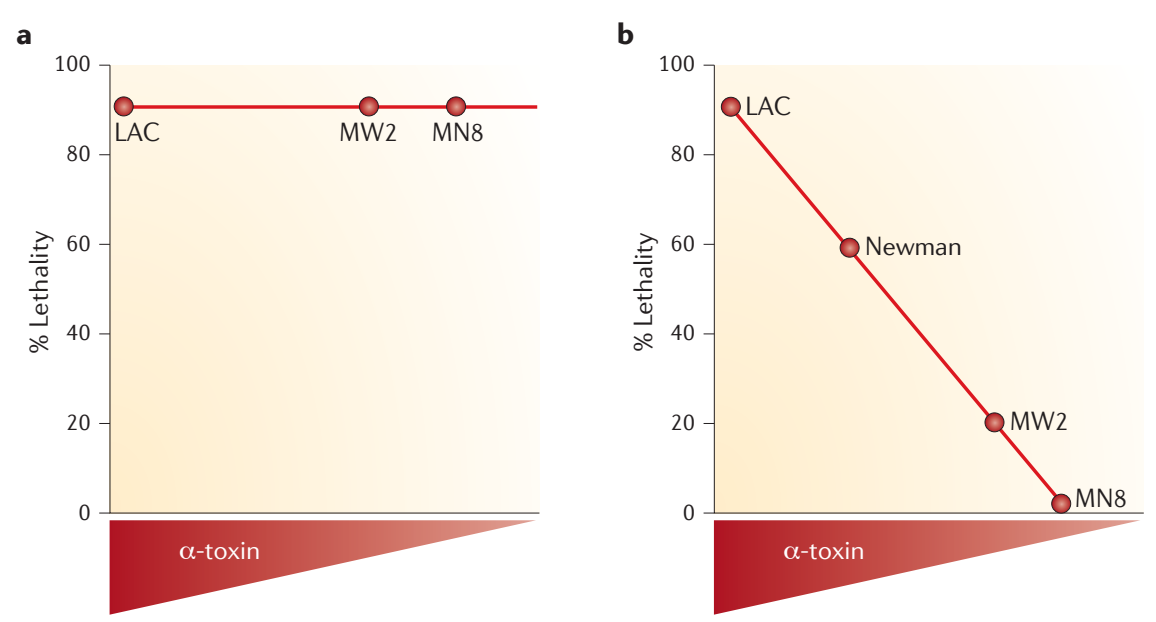

Figure 2 | Lethality induced by various Staphylococcus aureus strains as a function of the animal model used (mouse versus rabbit pneumonia model) and their level of $\alpha$-toxin production. a Inoculation of the S. aureus strains LAC, MW2 and MN8 at $6.5 \times 10^{8} \mathrm{CFUs} \mathrm{per} \mathrm{kg} \mathrm{in} \mathrm{the}$ rabbit pneumonia model shows that they are equally virulent 48 hours after infection. $\mathbf{b}$ | Inoculation of the S. aureus strains LAC, Newman, MW2 and MN8 at 6.5-10 × $10^{9} \mathrm{CFUs} \mathrm{per} \mathrm{kg} \mathrm{in} \mathrm{the} \mathrm{mouse} \mathrm{pneu-}$ monia model shows that virulence correlates with the level of $\alpha$-toxin production. Data taken from REFS 46,48,50,51.

seems to be hypervirulent (as it produces a-toxin at high levels, even though it makes SAgs at low levels). There are likely to be many additional reasons for the differential responses of mouse models compared with rabbit models and humans. However, it is clear that mouse resistance to SAgs and $\alpha$-toxin produces virulence outcomes that are not consistent with those that are observed in humans and rabbits. In our opinion, the reliance on mouse models has led to confusion and has perpetuated misconceptions in the staphylococcal field of research, including our understanding of the virulence of strains relative to one another, the role of various staphylococcal proteins in disease and the identification of staphylococcal molecules that have potential as human vaccine candidates. The identification of staphylococcal molecules that have potential as vaccine candidates is the most important consequence of the reliance on mouse models, and it may have made the most important contribution to the failure of $S$. aureus vaccine development.

\section{Basis for divergent responses}

The divergent responses of mice compared with humans and rabbits result from at least three major differences. First, the mouse transcriptional response to inflammatory stimuli (such as LPS) differs considerably from that of humans. Second, the effects of cytokines on the vasculature and the central nervous system in response to LPS and SAgs are different in mice compared with humans. Third, there are differences in the composition of the gut and vaginal microbiota in mice, rabbits and humans.

Transcriptional response: endotoxemia. It has been known since the early 1960s that mice are highly resistant to the effects of Gram-negative LPS ${ }^{56}$. A key recent study by Seok et al. investigated this difference at the molecular level and showed that mouse inflammatory responses to infectious agents in general, and to LPS specifically, are significantly different from those of humans ${ }^{21}$. Gene expression profiling of C57BL/6J mice and humans during endotoxemia showed a low correlation between the human genes and mouse orthologues and vice versa. In this study, the human response to endotoxemia was threefold greater than that of mice and was poorly predicted by the mouse model. A comparison of the human and mouse pathways that are activated or suppressed during endotoxemia showed that humans activate $\mathrm{Fc} \gamma$ receptor-mediated phagocytosis in macrophages and monocytes, interleukin-10 (IL-10) signalling, integrin signalling, B cell receptor signalling and Toll-like receptor signalling. In mice, these signalling pathways are poorly activated, particularly the Toll-like receptor signalling pathway $^{21}$. Most of the signalling pathways that are suppressed during endotoxemia in 
humans are activated in mice, particularly the calcium-induced $\mathrm{T}$ cell apoptosis pathway ${ }^{21}$. Similar results were obtained by analysis of the genomic response of humans and mice to other severe acute inflammatory diseases, including sepsis and acute respiratory distress syndrome. This study provides evidence that, at the molecular level, mouse models poorly reflect the molecular mechanisms of human inflammatory diseases.

Cytokine response: fever and hypotension. SAgs cause fevers and life-threatening illnesses in humans, owing to overstimulation of the immune system, which leads to the massive production of IL- $1 \beta$, IL- 6 , interferon- $\gamma$ (IFN $\gamma)$, tumour necrosis factor (TNF) and lymphotoxin- $\alpha^{57,58}$ (which is commonly known as a cytokine storm). Cytokines, such as IL- $1 \beta$ and IL-6, are endogenous pyrogens (most of the endogenous pyrogens that cause fevers in humans are primarily produced by immune cells) and subsequently initiate a fever cascade that begins with the production of prostaglandin E2 by the hypothalamus ${ }^{59,60}$. It has been known for decades that BALB/c mice do not respond to LPS with fever and instead show a dose-dependent reduction in body temperature $^{59}$, whereas even minute doses of LPS cause high fevers both in humans and in rabbits ${ }^{32,59}$. High fevers, such as those seen in patients with TSS and a variant form of TSS known as extreme pyrexia syndrome ${ }^{61}$, can participate in lethality.

The major pathway by which microorganisms that express LPS or SAgs cause fevers depends on interactions of these toxic molecules with immune cells via Toll-like receptors ${ }^{62,63}$ or SAg cross-bridging of $\mathrm{T}$ cell receptors and major histocompatibility complex class II (MHC II) molecules ${ }^{19}$, either peripherally or within the central nervous system ${ }^{59}$. As mentioned above, mice do not activate Toll-like receptor signalling in response to $\mathrm{LPS}^{21}$. It is also known that mouse $\mathrm{T}$ cells show decreased responses to SAgs, whereas both human and rabbit $\mathrm{T}$ cells (and antigen-presenting cells) respond comparably to SAgs ${ }^{33,56,64}$. The lack of susceptibility of mice to SAgs has led to attempts to humanize mice, particularly for MHC II (REFS 65-67). However, mice that are humanized for MHC II still do not respond to SAgs with all of the signs of TSS ${ }^{68}$.

Furthermore, massive production of TNF and lymphotoxin- $\alpha$ has major effects on the human and rabbit cardiovascular systems, which cause capillary leak, hypotension and shock. SAgs and $\alpha$-toxin (which, at high concentrations is cytotoxic but at low concentrations is highly pro-inflammatory ${ }^{35}$ ) have major roles in pneumonia, sepsis and infective endocarditis, and their effects are mediated by the induction of acute inflammation ${ }^{19,50,54}$. Together, these studies clearly indicate the differential production of proinflammatory cytokines by mice compared with rabbits and humans in response to either LPS or SAg stimulation.

Gut and vaginal microbiota. Interestingly, the susceptibility of humans and rabbits to SAgs correlates with the presence of Gramnegative bacteria that produce endotoxin shock-inducing LPS $^{69,70}$ (that is, E. coli in humans and Pasteurella multocida in rabbits) in the intestinal and vaginal tracts ${ }^{71-74}$. Rodents ${ }^{75}$ and non-human primates ${ }^{76}$ have fewer of these organisms. Young adult rabbits ( 3 months old) succumb to TSST1 at doses of $50 \mu \mathrm{g}$ per $\mathrm{kg}$ (REF. 39) and to LPS at $535 \mu \mathrm{g}$ per $\mathrm{kg}$ (REF. 32). At 8 months old, rabbits are colonized at a greater density by P. multocida and become 1,000 times more susceptible to LPS $\left(\mathrm{LD}_{100} 0.5 \mu \mathrm{g}\right.$ per $\mathrm{kg}$ ); concomitantly, the susceptibility of rabbits to the lethal effect of TSST1 increases 1,000 -fold $\left(\mathrm{LD}_{100} 0.05 \mu \mathrm{g}\right.$ per kg). Enhancement of LPS shock by TSST1 was shown in young adult rabbits that were administered TSST1 at increasing concentrations before LPS treatment ${ }^{32}$. TSST1 increases the susceptibility of rabbits to LPS lethality by up to $10^{6}$-fold ${ }^{32}$; this effect has been observed with all SAgs that have been tested ${ }^{32,77}$. The SAg-induced increase in LPS lethality results from impaired LPS clearance by the liver ${ }^{78}$, possibly via direct liver cytotoxicity and the synergistic effects of SAgs and LPS on the production of $\mathrm{TNF}^{33}$. Endotoxemia occurs in humans during TSS and in rabbits that have been injected with TSST1. This has led to the hypothesis that animals with a gut and vaginal microbiota that includes Gram-negative bacteria expressing LPS that is capable of inducing endotoxin shock are susceptible to SAgs plus LPS ${ }^{79,80}$, whereas animals such as rodents and non-human primates, in which Gram-negative bacteria that express toxic LPS are rarely detected, are resistant $^{76,81}$. Although synergism between TSST1 and LPS does occur in mice and rhesus macaques that have been injected with the toxins, the susceptibility to the SAg-LPS combination never reaches that of rabbits $^{33}$. However, it supports the idea that the resistance or susceptibility of mice and monkeys versus rabbits and humans results not only from differences in cardiovascular physiology but also from the presence of endogenous LPS and combined toxin effects on the immune system. On this basis, one would expect that mice could predict disease in non-human primates, whereas rabbits predict disease in humans.

\section{Vaccination is possible}

Targeting secreted virulence factors. Vaccination with purified SAgs or SAg toxoids alone or in combination with cytotoxins has proven to be a successful strategy to protect rabbits against lethal doses of USA100, USA200, USA300 and USA400 S. aureus clonal types and provides sterilizing immunity $^{82}$. Vaccination with TSST1, SEC or SEB protects rabbits from lethal challenge with CA-MRSA TSST $1^{+}$USA200, SEC $^{+}$USA400 or SEB ${ }^{+}$USA400 strains, respectively ${ }^{46}$. Three different TSST1 toxoids produce TSST1-neutralizing antibodies at high levels and protect rabbits from a TSST1 lethal challenge in an LPS enhancement model. A trivalent vaccine that is composed of TSST $1_{\mathrm{G} 31 \mathrm{~S}}$ (or TSST1 $1_{\mathrm{S} 32 \mathrm{P}}$ ), SEC and $\alpha$-toxin ${ }_{\mathrm{H} 35 \mathrm{~L}}$ provides complete protection against a lethal challenge with $S$. aureus $\mathrm{MNPE}$ (TSST1 ${ }^{+}, \mathrm{SEC}^{+}$ and $\alpha$-toxin ${ }^{\mathrm{HI}}$ ) in the rabbit pneumonia model ${ }^{47}$. Furthermore, a pentavalent vaccine that contains TSST1 $1_{\mathrm{G} 31 \mathrm{~S}}\left(\right.$ or TSST $\left.1_{\mathrm{S} 32 \mathrm{P}}\right), \mathrm{SEC}$, $\alpha$-toxin ${ }_{\mathrm{H} 35 \mathrm{~L}}, \beta$-toxin and $\gamma$-toxin protects rabbits from infective endocarditis and lethal sepsis when challenged with a lethal dose of $S$. aureus $\mathrm{MNPE}^{47}$. Studies in rabbits thus provide evidence that a multivalent vaccine targeting secreted staphylococcal virulence factors that are produced by nearly all S. aureus isolates protects against lifethreatening staphylococcal diseases ${ }^{82}$. These studies suggest that the neutralization of crucial SAgs and cytolysins effectively incapacitates $S$. aureus such that phagocytic elimination of the organism occurs, similarly to what would be expected for coagulasenegative staphylococci (coagulase-negative staphylococci produce fewer virulence factors than other $S$. aureus strains and are eliminated by innate immune mechanisms, such as the alternative complement cascade and neutrophil opsonization).

\section{Conclusions}

The virulence of many bacterial pathogens that cause major human diseases is often multifactorial. The diseases that these organisms cause are becoming increasingly difficult to treat and require new thinking in vaccination strategies. So far, researchers have typically used rodents - particularly mouse models - to study human diseases that are caused by these bacterial pathogens, not because mouse infections mimic human illnesses, but rather owing to costs, the 
availability of genetically defined mutants and pressure from granting and regulatory agencies and reviewers. It is noteworthy that the pressure from granting agencies is beginning to change; for example, the US National Institutes of Health (NIH) recently issued a programme announcement to develop alternative animal models ${ }^{83}$.

Understanding $S$. aureus pathogenesis is crucial for the development of new treatment and vaccine strategies. In our opinion, success in these areas will rely on researchers being willing to switch to using animal models with immune systems and cardiovascular physiologies that more closely resemble those of humans, as such models are more likely to be predictive of human disease. This switch must take place if the biomedical research community is to develop effective vaccines and immunotherapeutic agents to target microorganisms that are increasingly difficult to treat.

Wilmara Salgado-Pabón and Patrick M. Schlievert are at the Department of Microbiology, Carver College of Medicine, University of Iowa, lowa City, Iowa 52242, USA. Correspondence to P.M.S. e-mail:patrick-schlievert@uiowa.edu doi:10.1038/nrmicro3308 Published online 7 July 2014

1. Klevens, R. M et al. Invasive methicillin-resistant Staphylococcus aureus infections in the United States. JAMA 298, 1763-1771 (2007)

2. Dinges, M. M., Orwin, P. M. \& Schlievert, P. M. Exotoxins of Staphylococcus aureus. Clin. Microbiol. Rev. 13, 16-34 (2000)

3. Lowy, F. D. Staphylococcus aureus infections. N. Engl. J. Med. 339, 520-532 (1998)

4. Fowler, V. G. et al. Effect of an investigational vaccine for preventing Staphylococcus aureus infections after cardiothoracic surgery: a randomized trial. JAMA 309 1368-1378 (2013).

5. Projan, S. J., Nesin, M. \& Dunman, P. M Staphylococcal vaccines and immunotherapy: to dream the impossible dream? Curr. Opin. Pharmacol. 6, 473-479 (2006).

6. Garcia-Lara, J. \& Foster, S. J. Anti-Staphylococcus aureus immunotherapy: current status and prospects. Curr. Opin. Pharmacol. 9, 552-557 (2009).

7. Verkaik, N. J., van Wamel, W. J. \& van Belkum, A. Immunotherapeutic approaches against Staphylococcus aureus. Immunotherapy 3, 1063-1073 (2011).

8. Proctor, R. A. Challenges for a universal Staphylococcus aureus vaccine. Clin. Infect. Dis. $\mathbf{5 4}$ 1179-1186 (2012).

9. Rasigade, J. P. \& Vandenesch, F. Staphylococcus aureus: a pathogen with still unresolved issues. Infect Genet. Evol. 21, 510-514 (2014).

10. Schaffer, A. C. \& Lee, J. C. Vaccination and passive immunisation against Staphylococcus aureus. Int J. Antimicrob. Agents 32 (Suppl. 1), 71-78 (2008).

11. Bagnoli, F., Bertholet, S. \& Grandi, G. Inferring reasons for the failure of Staphylococcus aureus vaccines in clinical trials. Front. Cell. Infect. Microbiol. 2, 16 (2012)

12. Fowler, V. G. Jr \& Proctor, R. A. Where does a Staphylococcus aureus vaccine stand? Clin. Microbiol. Infect. 20 (Suppl. 5), 66-75 (2014).

13. Spellberg, B. \& Daum, R. Development of a vaccine against Staphylococcus aureus. Semin. Immunopathol. 34, 335-348 (2012).

14. Proctor, R. A. Is there a future for a Staphylococcus aureus vaccine? Vaccine 30, 2921-2927 (2012).

15. Skurnik, D. et al. Natural antibodies in normal human serum inhibit Staphylococcus aureus capsular polysaccharide vaccine efficacy. Clin. Infect. Dis. 55 1188-1197 (2012).
16. DeDent, A. Kim, H. K. Missiakas, D. \& Schneewind, O. Exploring Staphylococcus aureus pathways to disease for vaccine development. Semin Immunopathol. 34, 317-333 (2012).

17. Daum, R. S. \& Spellberg, B. Progress toward a Staphylococcus aureus vaccine. Clin. Infect. Dis. $\mathbf{5 4}$ 560-567 (2012)

18. Schlievert, P. M. Staphylococcal scarlet fever: role of pyrogenic exotoxins. Infect. Immun. 31, 732-736 (1981).

19. Spaulding, A. R. et al. Staphylococcal and streptococcal superantigen exotoxins. Clin. Microbiol. Rev. 26, 422-447 (2013).

20. Salgado-Pabón, W. et al. Superantigens are critical for Staphylococcus aureus infective endocarditis, sepsis, and acute kidney injury. mBio 4, e00494-13 (2013).

21. Seok, J. et al. Genomic responses in mouse models poorly mimic human inflammatory diseases. Proc. Natl Acad. Sci. USA 110, 3507-3512 (2013).

22. Duranthon, V. et al. On the emerging role of rabbit as human disease model and the instrumental role of novel transgenic tools. Transgen. Res. 21, 699-713 (2012).

23. Peng, $X$. Transgenic rabbit models for studying human cardiovascular diseases. Comp. Med. 62, 472-479 (2012).

24. Schlievert, P. M. Cytolysins, superantigens, and pneumonia due to community-associated methicillin resistant Staphylococcus aureus. J. Infect. Dis. 200 , 676-678 (2009).

25. Patti, J. M., Allen, B. L., McGavin, M. J. \& Hook, M. MSCRAMM-mediated adherence of microorganisms to host tissues. Annu. Rev. Microbiol. 48, 585-617 (1994).

26. Foster, T. J. \& Hook, M. Surface protein adhesins of Staphylococcus aureus. Trends Microbiol. 6, 484-488 (1998)

27. Schlievert, P. M., Chuang-Smith, O. N., Peterson, M. L., Cook, L. C. \& Dunny, G. M. Enterococcus faecalis endocarditis severity in rabbits is reduced by IgG Fabs interfering with aggregation substance. PLOS ONE 5, e13194 (2010).

28. Kreft, B., Marre, R., Schramm, U. \& Wirth, R. Aggregation substance of Enterococcus faecalis mediates adhesion to cultured renal tubular cells. Infect. Immun. 60, 25-30 (1992).

29. Schlievert, P. M., Dunny, G. M., Stoehr, J. A. \& Assimacopoulos, A. P. Aggregation and binding substances enhance pathogenicity in a rabbit model of Enterococcus faecalis endocarditis. Adv. Exp. Med. Biol. 418, 789-791 (1997).

30. Schlievert, P. M. et al. Aggregation and binding substances enhance pathogenicity in rabbit models of Enterococcus faecalis endocarditis. Infect. Immun. 66 218-223 (1998)

31. Sauter, C \& Wolfensberger, C. Interferon in human serum after injection of endotoxin. Lancet 2, 852-853 (1980)

32. Schlievert, P. M. Enhancement of host susceptibility to lethal endotoxin shock by staphylococcal pyrogenic exotoxin type C. Infect Immun. 36, 123-128 (1982).

33. Dinges, M. M. \& Schlievert, P. M. Comparative analysis of lipopolysaccharide-induced tumour necrosis factor alpha activity in serum and lethality in mice and rabbits pretreated with the staphylococcal superantigen toxic shock syndrome toxin 1. Infect. Immun. 69, 7169-7172 (2001)

34. Schaedler, R. W. \& Dubos, R. J. The susceptibility of mice to bacterial endotoxins. J. Exp. Med. 113, 559-570 (1961).

35. Bhakdi, S \& Tranum-Jensen, J. Alpha-toxin of Staphylococcus aureus. Microbiol. Rev. 55, 733-751 (1991)

36. Lin, Y. C. et al. Pro-inflammatory exoprotein characterization of toxic shock syndrome Staphylococcus aureus. Biochemistry 50, 7157-7167 (2011).

37. Giantonio, B. J. et al. Superantigen-based immunotherapy: a phase I trial of PNU-214565, a monoclonal antibody-staphylococcal enterotoxin A recombinant fusion protein, in advanced pancreatic and colorectal cancer. J. Clin. Oncol. 15, 1994-2007 (1997)

38. Larkin, S. M., Williams, D. N., Osterholm, M. T., Tofte, R. W. \& Posalaky, Z. Toxic shock syndrome: clinical, laboratory, and pathologic findings in nine fatal cases. Ann. Intern. Med. 96, 858-864 (1982).

39. Lee, P. K., Deringer, J. R., Kreiswirth, B. N., Novick, R. P. \& Schlievert, P. M. Fluid replacement protection of rabbits challenged subcutaneous with toxic shock syndrome toxins. Infect. Immun. $\mathbf{5 9}$ 879-884 (1991).
40. MacDonald, K. L. et al. Toxic shock syndrome. A newly recognized complication of influenza and influenzalike illness. JAMA 257, 1053-1058 (1987).

41. Reingold, A. L. et al. Toxic shock syndrome surveillance in the United States, 1980 to 1981. Ann. Intern. Med. 96, 875-880 (1982).

42. Bartlett, P. et al. Toxic shock syndrome associated with surgical wound infections. JAMA 247, 1448-1450 (1982).

43. Nienaber, J. J. et al. Methicillin-susceptible Staphylococcus aureus endocarditis isolates are associated with clonal complex 30 genotype and a distinct repertoire of enterotoxins and adhesins. J. Infect. Dis. 204, 704-713 (2011).

44. Spaulding, A. R. et al. Comparison of Staphylococcus aureus strains for ability to cause infective endocarditis and lethal sepsis in rabbits. Front. Cell. Infect. Microbiol. 2, 18 (2012)

45. Schlievert, P. M. \& Peterson, M. L. Glycerol monolaurate antibacterial activity in broth and biofilm cultures. PLOS ONE 7, e40350 (2012).

46. Strandberg, K. L. et al. Staphylococcal superantigens cause lethal pulmonary disease in rabbits. J. Infect. Dis. 202, 1690-1697 (2010)

47. Spaulding, A. R. et al. Immunity to Staphylococcus aureus secreted proteins protects rabbits from serious illnesses. Vaccine 30, 5099-5109 (2012).

48. DeLeo, F. R. et al. Molecular differentiation of historic phage-type 80/81 and contemporary epidemic Staphylococcus aureus. Proc. Natl Acad. Sci. USA 108, 18091-18096 (2011)

49. Tenover, F. C. \& Goering, R. V. Methicillin-resistant Staphylococcus aureus strain USA300: origin and epidemiology. J. Antimicrob. Chemother. 64, 441-446 (2009).

50. Bubeck Wardenburg, J., Bae, T., Otto, M., Deleo, F. R $\&$ Schneewind, O. Poring over pores: $\alpha$-haemolysin and Panton-Valentine leukocidin in Staphylococcus aureus pneumonia. Nature Med. 13, 1405-1406 (2007).

51. Wilson, G. J. et al. A novel core genome-encoded superantigen contributes to lethality of community associated MRSA necrotizing pneumonia. PLOS Pathog. 7, e1002271 (2011)

52. Voyich, J.M. et al. Is Panton-Valentine leukocidin the major virulence determinant in community-associated methicillin-resistant Staphylococcus aureus disease? J. Infect. Dis. 194, 1761-1770 (2006).

53. Schlievert, P. M., Osterholm, M. T., Kelly, J. A. \& Nishimura, R. D. Toxin and enzyme characterization of Staphylococcus aureus isolates from patients with and without toxic shock syndrome. Ann. Intern. Med. 96, 937-940 (1982)

54. Bubeck Wardenburg, J. \& Schneewind, O. Vaccine protection against Staphylococcus aureus pneumonia. J. Exp. Med. 205, 287-294 (2008).

55. Montgomery, C. P. et al. Comparison of virulence in community-associated methicillin-resistant Staphylococcus aureus pulsotypes USA300 and USA400 in a rat model of pneumonia. J. Infect. Dis. 198, 561-570 (2008).

56. Tomai, M. A., Schlievert, P. M. \& Kotb, M. Distinct T-cell receptor $V$ beta gene usage by human $T$ lymphocytes stimulated with the streptococcal pyrogenic exotoxins and pep M5 protein. Infect. Immun. 60, 701-705 (1992).

57. Kotzin, B. L., Leung, D. Y., Kappler, J. \& Marrack, P. Superantigens and their potential role in human disease. Adv. Immunol. 54, 99-166 (1993).

58. McCormick, J. K., Yarwood, J. M. \& Schlievert, P. M Toxic shock syndrome and bacterial superantigens: an update. Annu. Rev. Microbiol. 55, 77-104 (2001).

59. Schlievert, P. M. \& Watson, D. W. Group A streptococcal pyrogenic exotoxin: pyrogenicity, alteration of blood-brain barrier, and separation of sites for pyrogenicity and enhancement of lethal endotoxin shock. Infect. Immun. 21, 753-763 (1978).

60. Schlievert, P. M. \& Watson, D. W. Biogenic amine involvement in pyrogenicity and enhancement of lethal endotoxin shock by group A streptococcal pyrogenic exotoxin. Proc. Soc. Exp. Biol. Med. 162, 269-274 (1979).

61. Assimacopoulos, A. P., Strandberg, K. L., Rotschafer, J. H. \& Schlievert, P. M. Extreme pyrexia and rapid death due to Staphylococcus aureus infection: analysis of 2 cases. Clin. Infect. Dis. $\mathbf{4 8}$ 612-614 (2009)

62. Beutler, B. TLR4 as the mammalian endotoxin sensor. Curr. Top. Microbiol. Immunol. 270, 109-120 (2002).

63. Salomao, R. et al. TLR signalling pathway in patients with sepsis. Shock 30 (Suppl. 1), 73-77 (2008). 
64. Dinges, M. M. \& Schlievert, P. M. Role of T cells and gamma interferon during induction of hypersensitivity to lipopolysaccharide by toxic shock syndrome toxin in mice. Infect. Immun. 69, 1256-1264 (2001).

65. Rajagopalan, G. et al. Evaluating the role of HLA-DQ polymorphisms on immune response to bacterial superantigens using transgenic mice. Tissue Antigens 71, 135-145 (2008)

66. Rajagopalan, G., Sen, M. M. \& David, C. S. In vitro and in vivo evaluation of staphylococcal superantigen peptide antagonists. Infect. Immun. 72, 6733-6737 (2004).

67. Nooh, M. M., El-Gengehi, N., Kansal, R., David, C. S. \& Kotb, M. HLA transgenic mice provide evidence for a direct and dominant role of HLA class II variation in modulating the severity of streptococcal sepsis. J. Immunol. 178, 3076-3083 (2007).

68. Yeung, R. S. et al. Human CD4 and human major histocompatibility complex class II (DQ6) transgenic mice: supersensitivity to superantigen-induced septic shock. Eur. J. Immunol. 26, 1074-1082 (1996).

69. Lindberg, A. A., Weintraub, A., Zahringer, U. \& Rietschel, E. T. Structure-activity relationships in lipopolysaccharides of Bacteroides fragilis. Rev. Infect. Dis. 12 (Suppl. 2), 133-141 (1990).

70. Galanos, C. et al. Biological activities of lipid A complexed with bovine-serum albumin. Eur. J. Biochem. 31, 230-233 (1972).

71. Todar, K. E. coli: gastroenteritis, urinary tract infections and neonatal meningitis. Todar's Online Textbook of Bacteriology [online], http:// textbookofbacteriology.net/e.coli.html (2014).

72. DiGiacomo, R. F., Garlinghouse, L. E. Jr \& Van Hoosier, G. L. Jr Natural history of infection with Pasteurella multocida in rabbits. J. Am. Vet. Med. Assoc. 183, 1172-1175 (1983).

73. Schlievert, P. M., Nemeth, K. A., Davis, C. C., Peterson, M. L. \& Jones, B. E. Staphylococcus aureus exotoxins are present in vivo in tampons. Clin. Vaccine Immunol. 17, 722-727 (2010).

74. Pérez de Rozas, A. M. et al. in Proceedings of the 9th World Rabbit Congress, Verona, Italy, 10-13 June 2008, (eds Xicato, G., Trocino, A., Lukefahr, S. D.) 1041-1044 (Fondazione Iniziative Zooprofilattiche e Zootecniche, Brescia - Italy, 2008)

75. Le Roy, T. et al. Intestinal microbiota determines development of non-alcoholic fatty liver disease in mice. Gut 62, 1787-1794 (2013).

76. Schlievert, P. M. et al. Glycerol monolaurate does not alter rhesus macaque (Macaca mulatta) vaginal lactobacilli and is safe for chronic use. Antimicrob. Agents Chemother. 52, 4448-4454 (2008).

77. Kim, Y. B. \& Watson, D. W. A purified group A streptococcal pyrogenic exotoxin. Physiochemical and biological properties including the enhancement of susceptibility to endotoxin lethal shock. J. Exp. Med. 131, 611-622 (1970).

78. Schlievert, P. M., Bettin, K. M. \& Watson, D. W. Inhibition of ribonucleic acid synthesis by group $A$ streptococcal pyrogenic exotoxin. Infect. Immun. 27. 542-548 (1980)

79. Chow, A. W., Percival-Smith, R., Bartlett, K. H., Goldring, A. M. \& Morrison, B. J. Vaginal colonization with Escherichia coli in healthy women. Determination of relative risks by quantitative culture and multivariate statistical analysis. Am. J. Obstet. Gynecol. 154, 120-126 (1986).

80. Stone, R. L. \& Schlievert, P. M. Evidence for the involvement of endotoxin in toxic shock syndrome. J. Infect. Dis. 155, 682-689 (1987).
81. Pantoja-Feliciano, I. G. et al. Biphasic assembly of the murine intestinal microbiota during early development. ISME J. 7, 1112-1115 (2013).

82. Spaulding, A. R. et al. Vaccination against Staphylococcus aureus pneumonia. J. Infect. Dis. 209, 1955-1962 (2014).

83. US National Institutes of Health. Development and Characterization of Animal Models for Ageing Research (R21) [online], http://grants.nih.gov/grants/ guide/pa-files/PA-13-156.html (NIH, 2013).

84. Neuhaus, F. C. \& Baddiley, J. A continuum of anionic charge: structures and functions of D-alanyl-teichoic acids in Gram-positive bacteria. Microbiol. Mol. Biol. Rev. 67, 686-723 (2003).

85. Weisman, L. E. et al. Phase 1/2 double-blind, placebo-controlled, dose escalation, safety, and pharmacokinetic study of pagibaximab (BSYX-A110), an antistaphylococcal monoclonal antibody for the prevention of staphylococcal bloodstream infections, in very-low-birth-weight neonates. Antimicrob. Agents Chemother 53, 2879-2886 (2009).

86. Weisman, L. E. et al. A randomized study of a monoclonal antibody (pagibaximab) to prevent staphylococcal sepsis. Pediatrics 128, 271-279 (2011).

87. Scher, I. Safety and efficacy of Pagibaximab injection in very low birth weight neonates for prevention of staphylococcal sepsis. Clinical trials.gov, [online] http:// clinicaltrials.gov/ct2/show/results/NCT00646399?ter $\mathrm{m}=$ Pagibaximab\&rank $=1$ \&sect $=$ X6015 - evnt (2011).

88. Fattom, A. et al. Effect of conjugation methodology, carrier protein, and adjuvants on the immune response to Staphylococcus aureus capsular polysaccharides. Vaccine 13, 1288-1293 (1995).

89. Arbeit, R. D., Karakawa, W. W., Vann, W. F. \& Robbins, J. B. Predominance of two newly described capsular polysaccharide types among clinical isolates of Staphylococcus aureus. Diagn. Microbiol. Infect. Dis. 2, 85-91 (1984).

90. Hochkeppel, H. K. et al. Serotyping and electron microscopy studies of Staphylococcus aureus clinical isolates with monoclonal antibodies to capsular polysaccharide types 5 and 8. J. Clin. Microbiol. 25, 526-530 (1987)

91. Karakawa, W. W. et al. Method for the serological typing of the capsular polysaccharides of Staphylococcus aureus. J. Clin. Microbiol. 22 445-447 (1985)

92. Sompolinsky, D., Schwartz, D., Samra, Z. Steinmetz, J. \& Siegman-Igra, Y. Septicaemia with two distinct strains of Staphylococcus aureus and dwarf variants of both. Isr. J. Med. Sci. 21, 434-440 (1985).

93. Thakker, M., Park, J. S., Carey, V. \& Lee, J. C. Staphylococcus aureus serotype 5 capsular polysaccharide is antiphagocytic and enhances bacterial virulence in a murine bacteraemia model. Infect. Immun. 66, 5183-5189 (1998).

94. Fattom, A. I., Sarwar, J., Ortiz, A. \& Naso, R. A Staphylococcus aureus capsular polysaccharide (CP) vaccine and $\mathrm{CP}$-specific antibodies protect mice against bacterial challenge. Infect. Immun. $\mathbf{6 4}$ 1659-1665 (1996).

95. Shinefield, H. et al. Use of a Staphylococcus aureus conjugate vaccine in patients receiving haemodialysis. N. Engl. J. Med. 346, 491-496 (2002).

96. Fattom, A. et al. Safety and immunogenicity of a booster dose of Staphylococcus aureus types 5 and 8 capsular polysaccharide conjugate vaccine
(StaphVAX) in haemodialysis patients. Vaccine $\mathbf{2 3}$ 656-663 (2004)

97. Benjamin, D. K. et al. A blinded, randomized, multicenter study of an intravenous Staphylococcus aureus immune globulin. J. Perinatol. 26, 290-295 (2006).

98. Lee, J. C., Park, J. S., Shepherd, S. E., Carey, V. \& Fattom, A. Protective efficacy of antibodies to the Staphylococcus aureus type 5 capsular polysaccharide in a modified model of endocarditis in rats. Infect. Immun. 65, 4146-4151 (1997).

99. Rupp, M. E. et al. Phase II, randomized, multicenter, double-blind, placebo-controlled trial of a polyclonal anti-Staphylococcus aureus capsular polysaccharide immune globulin in treatment of Staphylococcus aureus bacteraemia. Antimicrob. Agents Chemother. 51, 4249-4254 (2007).

100. Josefsson, E., Hartford, O., O’Brien, L., Patti, J. M. \& Foster, T. Protection against experimental Staphylococcus aureus arthritis by vaccination with clumping factor $\mathrm{A}$, a novel virulence determinant. J. Infect. Dis. 184, 1572-1580 (2001).

101. Vernachio, J. et al. Anti-clumping factor $A$ immunoglobulin reduces the duration of methicillinresistant Staphylococcus aureus bacteraemia in an experimental model of infective endocarditis. Antimicrob. Agents Chemother. 47, 3400-3406 (2003).

102. Weems, J. J. Jr. et al. Phase II, randomized, doubleblind, multicenter study comparing the safety and pharmacokinetics of tefibazumab to placebo for treatment of Staphylococcus aureus bacteraemia. Antimicrob. Agents Chemother. 50, 2751-2755 (2006).

103. DeJonge, M. et al. Clinical trial of safety and efficacy of INH-A21 for the prevention of nosocomial staphylococcal bloodstream infection in premature infants. J. Pediatr. 151, 260-265 (2007).

104. Burnie, J. P. et al. Identification of an immunodominant $\mathrm{ABC}$ transporter in methicillinresistant Staphylococcus aureus infections. Infect. Immun. 68, 3200-3209 (2000)

105. Burnie, J. et al. Analysis of 42 cases of septicaemia caused by an epidemic strain of methicillin-resistan Staphylococcus aureus: evidence of resistance to vancomycin. Clin. Infect. Dis. 31, 684-689 (2000).

106. Ohlsen, K. \& Lorenz, U. Immunotherapeutic strategies to combat staphylococcal infections. Int. J. Med. Microbiol. 300, 402-410 (2010)

107. Torres, V. J., Pishchany, G., Humayun, M., Schneewind, O. \& Skaar, E. P. Staphylococcus aureus IsdB is a haemoglobin receptor required for haem iron utilization. J. Bacteriol. 188, 8421-8429 (2006).

108. Kuklin, N. A. et al. A novel Staphylococcus aureus vaccine: iron surface determinant $B$ induces rapid antibody responses in rhesus macaques and specific increased survival in a murine $S$. aureus sepsis model. Infect. Immun. 74, 2215-2223 (2006).

109. Ebert, T. et al. A fully human monoclonal antibody to Staphylococcus aureus iron regulated surface determinant $\mathrm{B}$ (IsdB) with functional activity in vitro and in vivo. Hum. Antibodies 19, 113-128 (2010).

110. Moustafa, M. et al. Phase lla study of the immunogenicity and safety of the novel Staphylococcus aureus vaccine V710 in adults with end-stage renal disease receiving haemodialysis. Clin. Vaccine Immunol. 19, 1509-1516 (2012).

Competing interests statement

The authors declare no competing interests. 